\title{
Effective Tactics for Getting an Equipment Grant
}

\author{
Kenneth A. Taylor
}

Institute of Molecular Biophysics, Department of Biological Science, Florida State University, Tallahassee, FL 32306-4380

The instructor for this tutorial has been writing NIH and NSF shared instrumentation grants for 33 years (success rate 100\%) and has been reviewing them for the NIH for 28 years, most recently in 2015. The tutorial will draw on this experience.

Acquiring and maintaining cutting edge instrumentation is critical for maintaining a competitive research program. There are several funding mechanisms for acquiring shared equipment including the NIH Shared Instrumentation and High End Instrumentation Grant Programs (S10) and the NSF Major Research Instrumentation Program (MRI). The chief difference between the NIH and NSF programs is the requirement for matching funds. NIH requires no matching funds, though such funds are allowed and would be necessary if the acquisition cost exceeded the maximum expense allowed. The NSF MRI program requires a cost share amounting to $30 \%$ of the total project cost for applications from Ph.D.granting institutions of higher education and for non-degree-granting organizations. Non-Ph.D.-granting institutions of higher education are exempt from cost-sharing and cannot include it. In addition, institutions may submit as many S10 applications as they desire, but the MRI program will accept no more than two applications from a single organization. NIH and NSF have different requirements for the number of users whose research would benefit from the instrumentation acquisition. For the NIH, there must be a minimum of three investigators funded by the NIH, there is no maximum and once the minimum of three is reached, additional users need not all be NIH funded. For the NSF, a PI and up to four co-PIs who will benefit from the instrument are required. They need not be NSF funded.

The strategy for a successful application to both the NIH and NSF programs are rather similar. Selection of the user group is important because their science will determine whether the review panel evaluates the instruments impact on their research. The strength of the need and impact are critical. Since these instruments are to service multiple users, a critical mass of investigators is a key requirement. The commitment from the host institution is important in several ways. NSF requires a cost share. Though $\mathrm{NIH}$ does not, they are also highly interested in the institutional commitment, but in a different way. $\mathrm{NIH}$ puts more emphasis on the institutions commitment to keeping the instrument running and the infrastructure within which the instrument will be operated. Is it to be placed into a core facility? How is the core facility maintained? Does the institution provide financial backup to even out income fluctuations over time even as use fluctuates over time?

There are several common mistakes that should be avoided when preparing an equipment grant application. These include:

(1) Insufficient documentation on presence and accessibility of comparable equipment in your geographical location

(2) Insufficient justification for instrument or its accessories

(3) Insufficient grant funding to support the maintenance of the instrument 
(4) Weak institutional support for long term maintenance

(5) Poor administrative plan

Figure 1. Flow Chart for Shared Instrument Grant Assembly

\section{Instrument Choice}

\section{Configuration}

\section{Location \& Administration}

\section{Financing \& Maintenance}

Inventory of Comparable Instruments \& Availability

\section{User Group}

\section{\& Research Projects}

\section{Use Distribution}

Oversight - External

Advisory Committee

\section{Letters of Support}

